\title{
Speed and Heading Control of an Unmanned Surface Vehicle Based on State Error PCH Principle
}

\author{
Chengxing Lv $\mathbb{D}$, ${ }^{1,2}$ Haisheng Yu $\mathbb{D},{ }^{1}$ Zhili Hua, $^{2}$ Lei Li $\mathbb{D}^{2},{ }^{2}$ and Jieru Chi $\mathbb{D}^{1}$ \\ ${ }^{1}$ College of Automation and Electrical Engineering, Qingdao University, Qingdao 266071, China \\ ${ }^{2}$ Institute of Oceanographic Instrumentation, Qilu University of Technology, Shandong Academy of Sciences, \\ Shandong Provincial Key Laboratory of Ocean Environmental Monitoring Technology, \\ National Engineering and Technological Research Center of Marine Monitoring Equipment, Qingdao 266001, China \\ Correspondence should be addressed to Haisheng Yu; yu.hs@163.com
}

Received 21 August 2017; Revised 7 December 2017; Accepted 19 December 2017; Published 18 January 2018

Academic Editor: George Tsiatas

Copyright (c) 2018 Chengxing Lv et al. This is an open access article distributed under the Creative Commons Attribution License, which permits unrestricted use, distribution, and reproduction in any medium, provided the original work is properly cited.

This paper proposes a novel nonlinear control scheme based on energy-shaping (ES) principle and state error port-controlled Hamiltonian (PCH) systems for unmanned surface vehicles (USV) system. The PCH model of three degrees of freedom for USV kinetics system is established. By the ES principle, interconnection assignment and damping injection method is applied to the speed and heading control of the closed-loop USV system to realize an overall stability of control mechanism. Simulation results show that the validity and stability of control algorithm can be satisfied with the performance in speed and heading tracking of which the high simplification and portability make it applicable to the various region.

\section{Introduction}

Unmanned Surface Vehicle (USV) is operated on the surface of the water without crew operation. The first appearance of USV can be traced back to World War II, in which they were developed for the purpose of military use. With the development of correlative technique, USV becomes widespread in areas both military and civilian such as Mine Countermeasures, Environmental Monitoring, Maritime Security, AntiSubmarine Warfare, Electronic Warfare, Surface Warfare, Special Operation Forces, and Maritime Interdiction Operation. USV has been widely used for special missions. Now, with the innovation in electric propulsion technology, the small high speed unmanned systems will have wide applications. The motion control problem of USV is attracting more and more attention from scholars all over the world [1].

The dynamic performance characteristics of USV have an important part in the development of the automatic system for motion control. It is still a problem in the field of both control theory and robotics to have high quality motion control for USV systems. And a group of international and domestic academics have devoted much of their research on the nonlinear control of such vehicles. According to the related literature, various designed controller approaches have been proposed like sliding mode control, adaptive control, backstepping control, cascaded control theory, fuzzy logic control, and so on. As discussed by Liu et al. [2] comprehensive reviews present recent progress of the control approaches from the points of applications, methodologies, and challenges. As to the adaptive control, an active mechanism for unmanned vehicles, Klinger et al. [3] implemented an adaptive algorithm with the modified backstepping surge controller which has been field tested. Sonnenburg [4] and Sonnenburg and Woolsey [5] direct a speed controller algorithm by backstepping and Lyapunov's direct method, which also has been tested by USV. Dong et al. [6] present a state feedback based backstepping control algorithm to address the speed and trajectory tracking problem. Sean Kragelund et al. [7] proposed three different adaptive speed controllers and a model reference adaptive controller of a floating turbine. The major solutions of trajectory tracking problem are the method of feedback linearization, backstepping approach, Lyapunov's direct method, cascade system method, robust control, sliding mode control, and so on. Some scholars used the backstepping approach and Lyapunov's direct method to resolve the trajectory tracking problem of the USV system 
$[8,9]$; the result showed that the controller can still force the trajectory. In paper [10], a sliding mode trajectory tracking controller was developed, and the result showed that the USV could track circular and straight line trajectory. Kahveci and Ioannou [11] proposed an adaptive law which is combined with a control design including a Linear Quadratic (LQ) controller to resolve the steering control for uncertain ship dynamic. However, the nonlinear control methods mentioned above are still not implemented because the process of control is too complex, and there is still not a comprehensive and practical control law which can be robust in vessel dynamics.

Recently, some scholars pay more attention to the portcontrolled Hamiltonian (PCH) theory and interconnection and damping assignment (IDA-PBC) method [12-15], and in the design of nonlinear control systems field the interconnection and damping assignment approach has gradually become a significant method. In this paper, our main objective is to develop a new speed and heading angle controller of USV which combined interconnection and damping assignment method and state error approach. The organization of this paper is as follows: mathematical PCH kinetics model of the USV is presented in Section 2, a detailed design of the controller is in Section 3, Section 4 analyzes the stability of the controller, and the simulation results are shown in Section 5.

\section{PCH Model of Unmanned Surface Vehicles}

2.1. The Model of USV. The structure of USV is shown in Figure 1. The propulsion system of USV is consisted of two propellers derived by two electric-powered motors. By the force and steering torque control, USV can keep moving in the condition of surge, sway, and yaw. Because, in this structure of three appreciable degrees of freedom, only two degrees can be actuated, USV under this structure is underactuated. In this context, dynamic model of USV has been extensively studied. In order to better facilitate the modeling design, USV is assumed to be moved in ideal fluid, and the mass is uniformly distributed. When building the reference frame, an origin of USV body coordinates coincides with the center of gravity, and both the center of gravity and buoyancy are perpendicular to the $Z$-axis. In physical design, USV is set to be port-starboard symmetrical; hence surge subsystem and sway-yaw subsystem are essentially decoupled [2].

From a physical standpoint, we should consider the impact of the nonlinear hydrodynamic damping in kinetics model to cover the applications from high speed to low speed. USV is assumed to be moved in ideal fluid, and the mass is uniformly distributed, so the uncertainties and disturbances are linear with velocity or slowly varying relative to the USV dynamics. Based on all of these above assumptions, the kinetics model $[3,16-21]$ of USV can be obtained as

$$
\begin{aligned}
m_{11} \dot{u}-m_{22} v r+d_{11} u & =f_{p}, \\
m_{22} \dot{v}+m_{11} u r+d_{22} v & =0, \\
m_{33} \dot{r}+\left(m_{22}-m_{11}\right) u v+d_{33} r & =T_{s} .
\end{aligned}
$$

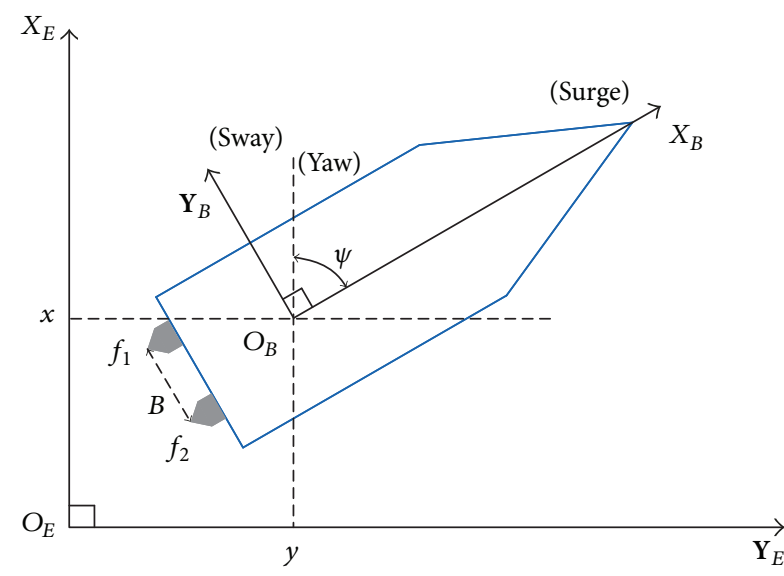

FIgURE 1: The motion coordinate system for USV.

The Kinematics model of heading subsystem of USV can be obtained as

$$
\dot{\psi}=r
$$

where $u$ is surge velocity, $v$ is sway velocity, and $r$ is yaw rate in body fixed reference frame. $m_{i i}$ are inertia coefficients of USV including mass effects added, $d_{i i}$ are hydrodynamic damping coefficients in conditions of surge, sway, and yaw, $f_{p}$ are the forces of propulsion system, and $T_{s}$ is the steering torque. $\psi$ denotes orientation angle of the vessel.

Then (1) can be expressed in matrix form:

$$
M \dot{v}+C(v) v+D v=u_{s} .
$$

Here $v=[u, v, r]^{T}, u_{s}=\left[f_{p}, 0, T_{s}\right]^{T}, M=$ $\operatorname{diag}\left\{m_{11}, m_{22}, m_{33}\right\} C(v)=\left[\begin{array}{ccc}0 & 0 & -m_{22} v \\ 0 & 0 & m_{11} u \\ m_{22} v & -m_{11} u & 0\end{array}\right]$, and $D=\operatorname{diag}\left\{d_{11}, d_{22}, d_{33}\right\}$.

$M$ is an inertia parameters matrix including the added body mass, $C(v)$ is the so-called Coriolis and centripetal matrix, and $D$ is hydrodynamic damping matrix.

The thrust forces $f_{p}$ and steering torque $T_{s}$ are functions of the two surge control thrust forces which are from each propeller:

$$
\begin{aligned}
& f_{p}=f_{1}+f_{2}, \\
& T_{s}=\frac{B\left(f_{1}-f_{2}\right)}{2},
\end{aligned}
$$

where $f_{1}$ is the thrust force which is produced by the first motor and $f_{2}$ is the thrust force which is produced by the second motor. $B$ is the distance between the propellers. From (4), the thrust allocated to each propeller, $f_{1}$ and $f_{2}$, can be calculated as

$$
\begin{aligned}
& f_{1}=\frac{f_{p}}{2}+\frac{T_{s}}{B}, \\
& f_{2}=\frac{f_{p}}{2}-\frac{T_{s}}{B} .
\end{aligned}
$$


2.2. PCH Systems. The general form for a nonlinear dynamical system can be shown as follows:

$$
\begin{aligned}
& \dot{x}=f(x)+g(x) u_{s}, \\
& y=h(x),
\end{aligned}
$$

where $x \in \mathbb{R}^{n}$ is the state vector, $y \in \mathbb{R}^{m}$ is the output vector, and $u_{s} \in \mathbb{R}^{m}$ is the input vector. From $[13,14]$ we can see that if there is possible way to find a nonnegative function $V(x)(V(0)=0)$ such that

$$
V(x(t))-V(x(0)) \leq \int_{0}^{t} y^{T}(\tau) u_{s}(\tau) d \tau
$$

the system described by (6) is passive. Then, the PCH system with dissipation can be described as follows [14]:

$$
\begin{aligned}
& \dot{x}=[J(x)-D(x)] \frac{\partial H(x)}{\partial x}+g(x) u_{s}, \\
& y=g^{T}(x) \frac{\partial H(x)}{\partial x}
\end{aligned}
$$

where $D(x)$ is positive semidefinite symmetric matrix and $D(x)=D^{T}(x) \geq 0$. It represents the dissipation of the system. The interconnection structure of the system is represented by the skew-symmetric matrix $J(x)=-J^{T}(x)$ and matrix $g(x)$. $H(x)$ is the Hamiltonian function which defines the stored energy function of the system.

The variation of internal energy of the dynamical system (8) equals the power which was provided with the system by the environment plus the dissipated power. The PCH system (8) model's energy balance equation is as follows:

$$
\begin{aligned}
\frac{d H(x)}{d t} & =\left[\frac{\partial H(x)}{\partial x}\right]^{T} \dot{x} \\
& =y^{T} u_{s}-\left[\frac{\partial H(x)}{\partial x}\right]^{T} D(x)\left[\frac{\partial H(x)}{\partial x}\right] \\
& \leq y^{T} u_{s} .
\end{aligned}
$$

In time-interval $[0, t],(9)$ establishes the passivity properties of the PCH system. which is the same as (7):

$$
H(x(t))-H(x(0)) \leq \int_{0}^{t} y^{T}(\tau) u_{s}(\tau) d \tau .
$$

2.3. PCH Model of USV Kinetics System. From the system described by (3) and (6), the state vector and the input vector of the system are defined as follows:

$$
\begin{gathered}
x=\left[\begin{array}{l}
x_{1} \\
x_{2} \\
x_{3}
\end{array}\right]=\left[\begin{array}{l}
m_{11} u \\
m_{22} v \\
m_{33} r
\end{array}\right], \\
u_{s}=\left[\begin{array}{l}
u_{1} \\
u_{2} \\
u_{3}
\end{array}\right]=\left[\begin{array}{c}
f_{p} \\
0 \\
T_{s}
\end{array}\right] .
\end{gathered}
$$

The Hamiltonian function of the USV dynamic system can be defined as

$$
H(x)=\frac{1}{2} x^{T} M^{-1} x=\frac{1}{2}\left(m_{11} u^{2}+m_{22} v^{2}+m_{33} r^{2}\right) .
$$

Combine (3), (8), and (12); then the PCH model of USV dynamic system can be obtained as follows:

$$
\begin{aligned}
\dot{x}= & {\left[\left(\begin{array}{ccc}
0 & 0 & m_{22} v \\
0 & 0 & -m_{11} u \\
-m_{22} v & m_{11} u & 0
\end{array}\right)\right.} \\
& \left.-\left(\begin{array}{ccc}
d_{11} & 0 & 0 \\
0 & d_{22} & 0 \\
0 & 0 & d_{33}
\end{array}\right)\right] \frac{\partial H}{\partial x}+\left(\begin{array}{lll}
1 & 0 & 0 \\
0 & 1 & 0 \\
0 & 0 & 1
\end{array}\right) u_{s},
\end{aligned}
$$

where

$$
\begin{aligned}
& J(x)=\left[\begin{array}{ccc}
0 & 0 & m_{22} v \\
0 & 0 & -m_{11} u \\
-m_{22} v & m_{11} u & 0
\end{array}\right], \\
& D(x)=\left[\begin{array}{ccc}
d_{11} & 0 & 0 \\
0 & d_{22} & 0 \\
0 & 0 & d_{33}
\end{array}\right] \\
& g(x)=\left[\begin{array}{lll}
1 & 0 & 0 \\
0 & 1 & 0 \\
0 & 0 & 1
\end{array}\right] \\
& \frac{\partial H}{\partial x}=M^{-1} x=\left[\begin{array}{l}
u \\
v \\
r
\end{array}\right]
\end{aligned}
$$

\section{The Controller Design of Speed and Heading}

In the design of energy controller, energy optimizing is realized by port-controlled Hamiltonian model. As to the PCH system described by (8), how to obtain a feedback defined by (17) that can keep the closed-loop system stable is the key point.

Assuming $x_{*}$ is a desired equilibrium, then the state error will be $\tilde{x}=x-x_{*}$. The final objective of IDA-PBC [12] is to find $\beta(x), J_{a}, D_{a}$ matching the condition

$$
\begin{aligned}
J_{d}(\tilde{x}) & =J(\tilde{x})+J_{a}=-J_{d}^{T}(\tilde{x}), \\
D_{d}(\tilde{x}) & =D(\tilde{x})+D_{a}=D_{d}^{T}(\tilde{x}) \geq 0, \\
u_{s} & =\beta(x) .
\end{aligned}
$$

Then the closed-loop system (8) follows a state error $\mathrm{PCH}$ form:

$$
\dot{\tilde{x}}=\left[J_{d}(\widetilde{x})-D_{d}(\widetilde{x})\right] \frac{\partial H_{d}}{\partial \tilde{x}} .
$$


The desired Hamilton function is chosen as

$$
H_{d}(\tilde{x})=\frac{1}{2}\left[m_{11}\left(u-u_{*}\right)^{2}+m_{22} v^{2}+m_{33} r^{2}\right] .
$$

Then we choose

$$
\begin{gathered}
J_{a}=\left[\begin{array}{ccc}
0 & -J_{12} & J_{13} \\
J_{12} & 0 & J_{23} \\
-J_{13} & -J_{23} & 0
\end{array}\right], \\
D_{a}=\left[\begin{array}{ccc}
d_{a 1} & 0 & 0 \\
0 & d_{a 2} & 0 \\
0 & 0 & d_{a 3}
\end{array}\right],
\end{gathered}
$$

where $J_{12}, J_{13}, J_{23}$, and $d_{a 1}, d_{a 2}, d_{a 3}$ are the designed parameters.

Consider the closed-loop system (18) with feedback control by (17); substituting $x=\tilde{x}+x_{*}$ into PCH system (8), we get

$$
\begin{aligned}
\dot{\tilde{x}}= & {\left[J\left(\tilde{x}+x_{*}\right)-D\right] M^{-1}\left(\tilde{x}+x_{*}\right) } \\
& +g\left(\tilde{x}+x_{*}\right) \beta(x)-\dot{x}_{*} .
\end{aligned}
$$

In USV PCH structure model, we could get the hydrodynamic damping matrix as constant matrix. So we used the symbol $D$ to represent the symbol $D(x)$.

From the PCH system (8), we can derive

$$
\dot{x}_{*}=\left[J\left(x_{*}\right)-D\right] M^{-1} x_{*}+g\left(x_{*}\right) u_{s *},
$$

where $u_{s *}$ is the input vector which corresponds to coming to the equilibrium point in (1).

If the condition (23) can be set up,

$$
J\left(\tilde{x}+x_{*}\right)=J(\tilde{x})+J\left(x_{*}\right),
$$

then substitute formulas (22) and (23) into (21); the state error model can be obtained by

$$
\begin{aligned}
\dot{\tilde{x}}= & {[J(\tilde{x})-D] M^{-1} \tilde{x}+J\left(x_{*}\right) M^{-1} \tilde{x}+J(\tilde{x}) M^{-1} x_{*} } \\
& +g\left(\tilde{x}+x_{*}\right) \beta(x)-g\left(x_{*}\right) u_{s *} .
\end{aligned}
$$

According to (16) and (17), the above formula can be written as (18), so the feedback control can be obtained by

$$
\begin{aligned}
g(x) \beta(x)= & {\left[J_{a}-D_{a}-J\left(x_{*}\right)\right] M^{-1} \tilde{x}-J(\tilde{x}) M^{-1} x_{*} } \\
& +g\left(x_{*}\right) u_{s *} .
\end{aligned}
$$

We define $u_{*}$ and $\psi_{*}$, respectively, referring to the desired surge speed and yaw angle; then the state $[u, v, r, \psi]^{T}$ is globally uniformly asymptotically convergent to $\left[u_{*}, 0,0, \psi_{*}\right]^{T}$ [22]. So the equilibrium point of $x_{*}$ will be $\left[m_{11} u_{*}, 0,0\right]^{T}$. When the system is coming to the equilibrium point, from (1) we can obtain

$$
u_{s *}=\left[\begin{array}{c}
d_{11} u_{*} \\
0 \\
0
\end{array}\right] .
$$

Substituting (16), (20), and (26) into the above formula (25), the energy controller becomes

$$
\begin{aligned}
f_{p}= & J_{13} r-J_{12} v-d_{a 1}\left(u-u_{*}\right)+d_{11} u_{*}, \\
0= & J_{12}\left(u-u_{*}\right)+J_{23} r-d_{a 2} v+m_{11} u_{*} r, \\
T_{s 1}= & u_{*} m_{22} v-\left(J_{23}+m_{11} u_{*}\right) v-J_{13}\left(u-u_{*}\right) \\
& -d_{a 3} r .
\end{aligned}
$$

Let $J_{13}=m_{22} v, J_{23}=-m_{11} u$, and $J_{12}=m_{33} r$; then the energy controller further becomes

$$
\begin{aligned}
f_{p} & =\left(m_{22}-m_{33}\right) v r-d_{a 1}\left(u-u_{*}\right)+d_{11} u_{*}, \\
0 & =\left(m_{33}-m_{11}\right)\left(u-u_{*}\right) r-d_{a 2} v, \\
T_{s} & =\left(m_{11}-m_{22}\right)\left(u-u_{*}\right) v+m_{22} v u_{*}-d_{a 3} r .
\end{aligned}
$$

As to the controller development of heading, the state error method is taken as the feedback control law for the yaw subsystem. The yaw subsystem of motion is given by (1) and (2) can be rewritten as

$$
\begin{aligned}
m_{33} \dot{r}+\left(m_{22}-m_{11}\right) u v+d_{33} r & =T_{s}, \\
\dot{\psi} & =r .
\end{aligned}
$$

Which is typical of cascade control system. If the heading tracking error is defined as

$$
e_{\psi}=\left(\psi-\psi_{*}\right)
$$

where $\psi_{*}$ is the desired heading angle, and the selected linearization control is in the form of $\dot{\psi}=\dot{\psi}_{*}-k_{\psi} e_{\psi}$, then the heading tracking error dynamics will be

$$
\dot{e}_{\psi}+k_{\psi} e_{\psi}=0 \text {. }
$$

When the values of the surge velocity gain $k_{\psi}$ are positive, the error dynamics for $e_{\psi}$ would remain stable. The error dynamics controller will be $T_{s 2}=-k_{\psi} e_{\psi}$. Combining the equation mentioned before with the third formula of (28), then the heading controller is

$$
\begin{aligned}
T_{s} & =T_{s 1}+T_{s 2} \\
& =\left(m_{11}-m_{22}\right)\left(u-u_{*}\right) v+m_{22} v u_{*}-d_{a 3} r-k_{\psi} e_{\psi} .
\end{aligned}
$$

It is obvious that the surge velocity always couples with yaw rate, as a result of which it is impossible to control either one of them independently. Checking the sway velocity subsystem which is the second formula of (1),

$$
m_{22} \dot{v}+m_{11} u r+d_{22} v=0 .
$$

By using the control law of heading angle and surge speed, we can see that the sway subsystem in (33) reduces to

$$
m_{22} \dot{v}=-m_{11} u_{*} \dot{\psi}_{*}-d_{22} v \text {. }
$$

When the time $t \rightarrow \infty$, finally the sway velocity always has $v \rightarrow 0$. That means the sway velocity $v$ is exponentially stable for the case of $\dot{\psi}_{*}=0$. 
From all the above analysis of the controller, the combined surge speed and heading controller are taken as

$$
\begin{aligned}
f_{p}= & \left(m_{22}-m_{33}\right) v r-d_{a 1}\left(u-u_{*}\right)+d_{11} u_{*}, \\
T_{s}= & \left(m_{11}-m_{22}\right)\left(u-u_{*}\right) v+m_{22} v u_{*}-d_{a 3} r \\
& -k_{\psi} e_{\psi} .
\end{aligned}
$$

\section{The Stability of the Controller}

We will analyze the stability of the energy controller in this section. Liao et al. [22] pointed that the combined surge speed and heading control laws make the state $[u, v, r, \psi]^{T}$ globally uniformly asymptotically convergent to $\left[u_{*}, 0,0, \psi_{*}\right]^{T}$ and bounded. Here we consider the Lyapunov function of the close-loop system defined as

$$
V_{e}=H_{d}(\widetilde{x})+\frac{1}{2} k_{\psi} e_{\psi}^{2}
$$

We can compute the time derivative of $V_{e}$ with respect to time along the solutions of the close-loop system

$$
\begin{aligned}
\dot{V}_{e} & =\frac{d H_{d}(\tilde{x})}{d t}+k_{\psi} e_{\psi} \dot{e}_{\psi}=\left[\frac{\partial H_{d}(\tilde{x})}{\partial \tilde{x}}\right]^{T} \dot{\tilde{x}}-k_{\psi}{ }^{2} e_{\psi}{ }^{2} \\
& =\left[\frac{\partial H_{d}(\tilde{x})}{\partial \tilde{x}}\right]^{T}\left[J_{d}(\tilde{x})-D_{d}(\tilde{x})\right] \frac{\partial H_{d}}{\partial \tilde{x}}-k_{\psi}{ }^{2} e_{\psi}{ }^{2} .
\end{aligned}
$$

As $J_{d}(\tilde{x})$ is the skew-symmetric matrix, we can obtain

$$
\left[\frac{\partial H_{d}(\tilde{x})}{\partial \tilde{x}}\right]^{T} J_{d}(\tilde{x}) \frac{\partial H_{d}}{\partial \tilde{x}}=0 .
$$

According to $D_{d}(\tilde{x})$ being positive semidefinite symmetric matrix, hence

$$
-\left[\frac{\partial H_{d}(\tilde{x})}{\partial \tilde{x}}\right]^{T} D_{d}(\tilde{x}) \frac{\partial H_{d}}{\partial \tilde{x}} \leq 0 .
$$

Obviously, we can get $V_{e}$ is positive definite and $\dot{V}_{e}$ is negative semidefinite. By using the Lyapunov stability theory, the closed-loop system establishes stability. So $\left[u_{*}, 0,0, \psi_{*}\right]^{T}$ is taken as the equilibrium of the closed-loop system. Additionally, if the largest invariant set of system equals $\{0\}$, the system is asymptotically stable.

From (14), we can get that the structures of the matrices $J(x)$ and $D(x)$ are maintained if the parameters are with uncertain displacement and drag. $J(x)$ is also positive semidefinite symmetric matrix, and $J(x)=-J^{T}(x) . D(x)$ is also positive semidefinite symmetric matrix, and $D(x)=$ $D^{T}(x) \geq 0$. Because the interconnection and damping structures of the system remain unchanged, the stability of the system is also asymptotically stable.

\section{System Simulation}

The numerical simulation is performed by MATLAB/ Simulink. USV for modeling has a length of $1.2 \mathrm{~m}$ and a mass of $17.5 \mathrm{~kg}$, and two DC motors were equipped for providing surge force and yaw moment by driving two propellers. The ship model's parameters are calculated [23]:

$$
\begin{aligned}
& m_{11}=25.8 \mathrm{~kg}, \\
& m_{22}=33.8 \mathrm{~kg}, \\
& m_{33}=2.76 \mathrm{~kg} \cdot \mathrm{m}^{2}, \\
& r_{11}=12 \mathrm{~kg} / \mathrm{s}, \\
& r_{22}=17 \mathrm{~kg} / \mathrm{s}, \\
& r_{33}=0.5 \mathrm{~kg} \cdot \mathrm{m}^{2} / \mathrm{s} .
\end{aligned}
$$

From the stability analysis of the controller, the parameters should be $d_{a 1}, d_{a 3} \geq 0, k_{\psi} \geq 0$. And the controller parameters can be ranged through the spectrum. Figure 2 gives the surge speed responses of different damping parameters $\left(d_{a 1}=100\right.$, $d_{a 1}=2000$, and $\left.d_{a 1}=4000\right)$. From Figure 2 , we can know that the surge speed response has better performance when $d_{a 1}=2000$. Figure 3 gives the heading angle responses of different parameters $\left(d_{a 3}=100, k_{\psi}=500, d_{a 3}=500\right.$, $k_{\psi}=2000, d_{a 3}=1000$, and $\left.k_{\psi}=3000\right)$. We can know that the heading angle response has better performance when $d_{a 3}=500, k_{\psi}=2000$. So the design parameters are chosen as $d_{a 1}=2000, d_{a 3}=500$, and $k_{\psi}=2000$. The desired speed and heading references are $\left[u_{*}, 0,0, \psi_{*}\right]^{T}=[1,0,0,0.5]^{T}$. At the moment of $t=10 \mathrm{~s}$, load disturbances $\Delta \xi_{f_{p}}=10 \mathrm{~N}$ and $\Delta \xi_{T_{s}}=10 \mathrm{Nm}$ are added separately to the system, and duration of the disturbances added to the system is $1 \mathrm{~s}$. Dynamic response and disturbance attenuation of the control system are, respectively, shown in Figures 4 and 5, from which the satisfactory results are obtained by methods mentioned in Section 3. At the moment of $t=10 \mathrm{~s}$, the desired surge speed and heading angle are set to $\left[u_{*}, 0,0, \psi_{*}\right]^{T}=[1.3,0,0,1]^{T}$, respectively. Figures 6 and 7 are shown where the proposed control approach has extremely quick tracking performance.

Figure 8 shows that the state $[u, v, r, \psi]^{T}$ is globally uniformly asymptotically convergent to $\left[u_{*}, 0,0, \psi_{*}\right]^{T}$. When the system is coming to the equilibrium point, the sway velocity $v$ and the yaw rate $r$ always tend to Zero.

We use the classical PID speed and heading controller which is shown in Figure 9 to compare with the proposed control approach. Figure 10 gives the surge speed responses of different parameters $\left(k_{p}=500, k_{i}=10, k_{d}=100 ; k_{p}=1000\right.$, $k_{i}=10, k_{d}=100 ; k_{p}=5000, k_{i}=10$, and $\left.k_{d}=100\right)$. We can know that the surge speed response has better performance when $k_{p}=5000, k_{i}=10$, and $k_{d}=100$. Figure 11 gives heading angle responses of different parameters $\left(k_{p}=200\right.$, $k_{i}=0, k_{d}=100 ; k_{p}=1000, k_{i}=0, k_{d}=300 ; k_{p}=2000$, $k_{i}=0$, and $\left.k_{d}=500\right)$. We can know that the heading angle response has better performance when $k_{p}=2000$, $k_{i}=0$, and $k_{d}=500$. Figures 12 and 13 are shown where the proposed control approach has tracking performance similar to the classical PID approach. Figures 14 and 15 show the surge speed and heading angle responses when the load 


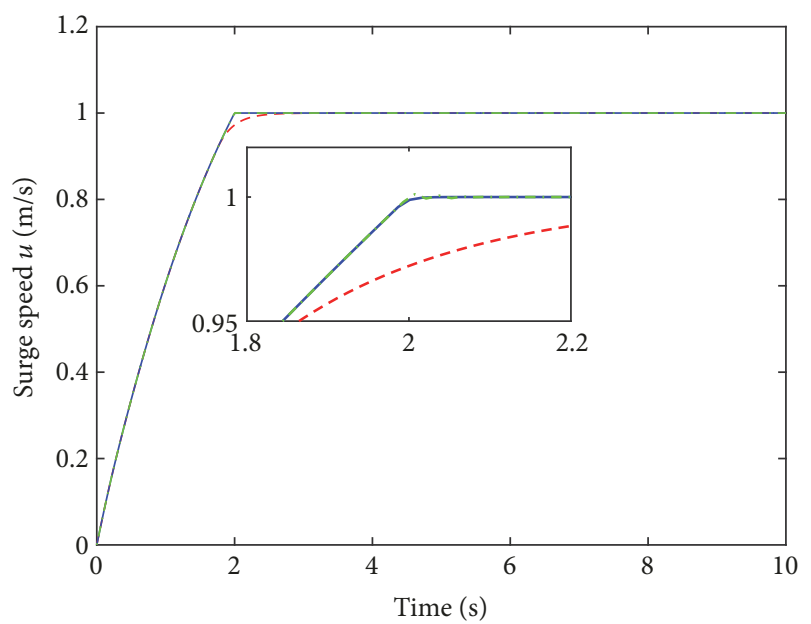

$$
\begin{aligned}
--d_{a 1} & =100 \\
- & d_{a 1}=2000 \\
--d_{a 1} & =4000
\end{aligned}
$$

FIGURE 2: Surge speed curves with different parameters.

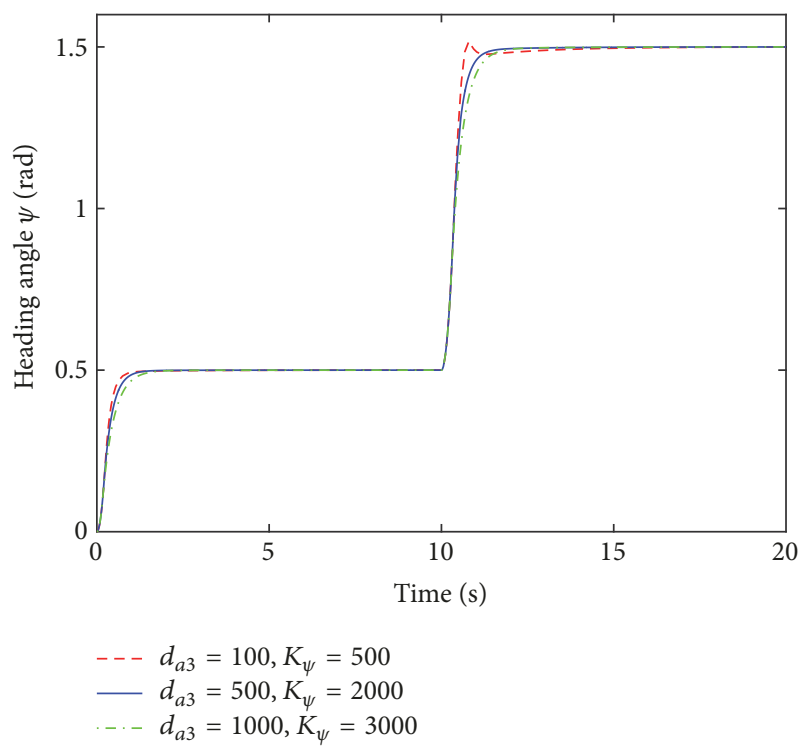

Figure 3: Heading angle curves with different parameters.

disturbances $\Delta \xi_{f_{p}}=10 \mathrm{~N}$ and $\Delta \xi_{T_{s}}=10 \mathrm{Nm}$ are added separately to the different controllers at $t=10 \mathrm{~s}$. From Figures 14 and 15 , we can see that the state error PCH controller has load disturbances attenuation performance similar to the classical PID controller.

The above simulation results show that the proposed control approach has good performance in dynamic and steady state. From the simulation results shown above, the proposed control method could to some extent achieve a better performance for the signal tracking of the given speed and heading angle.

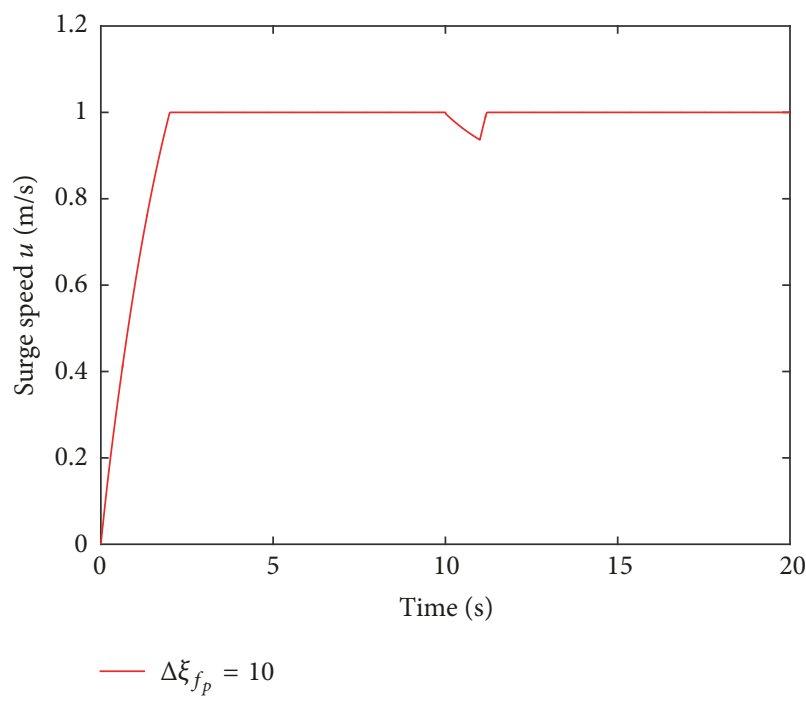

FIgURE 4: Surge speed curve of USV.

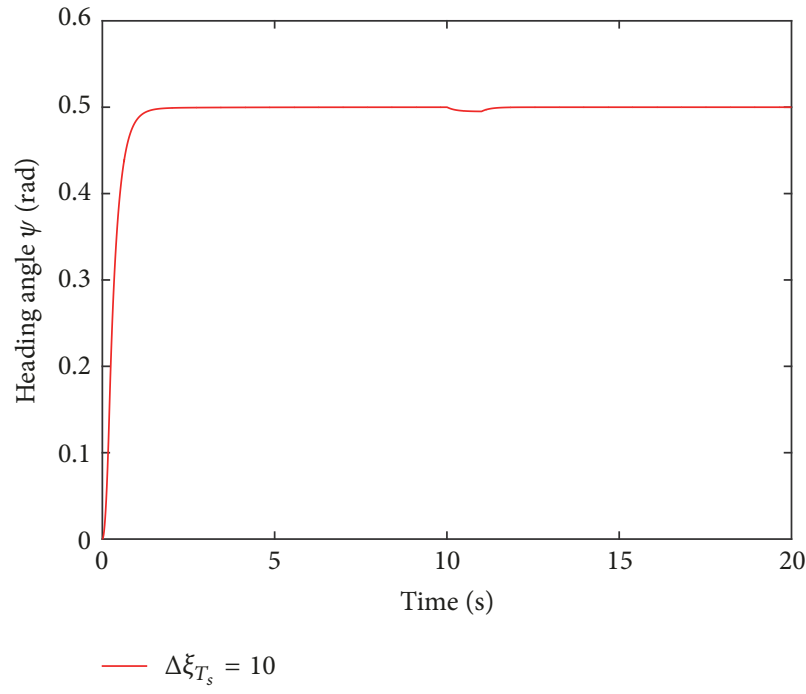

FIGURE 5: Heading angle curve of USV.

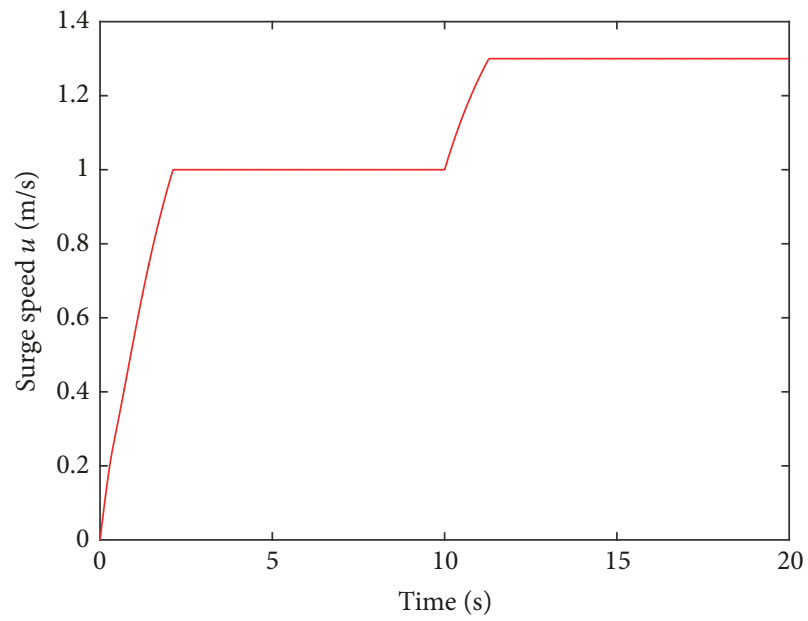

FIGURE 6: Surge speed tracking curve. 


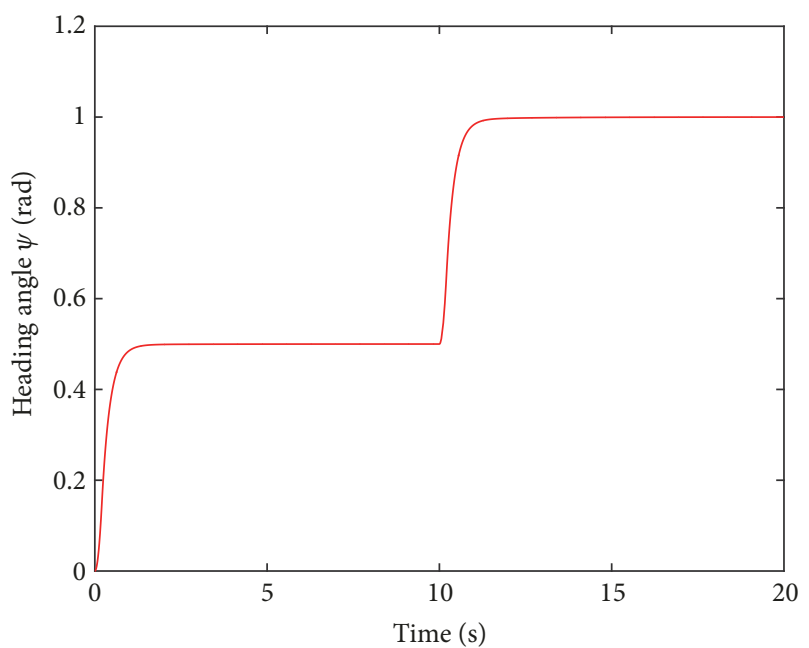

Figure 7: Heading angle tracking curve.

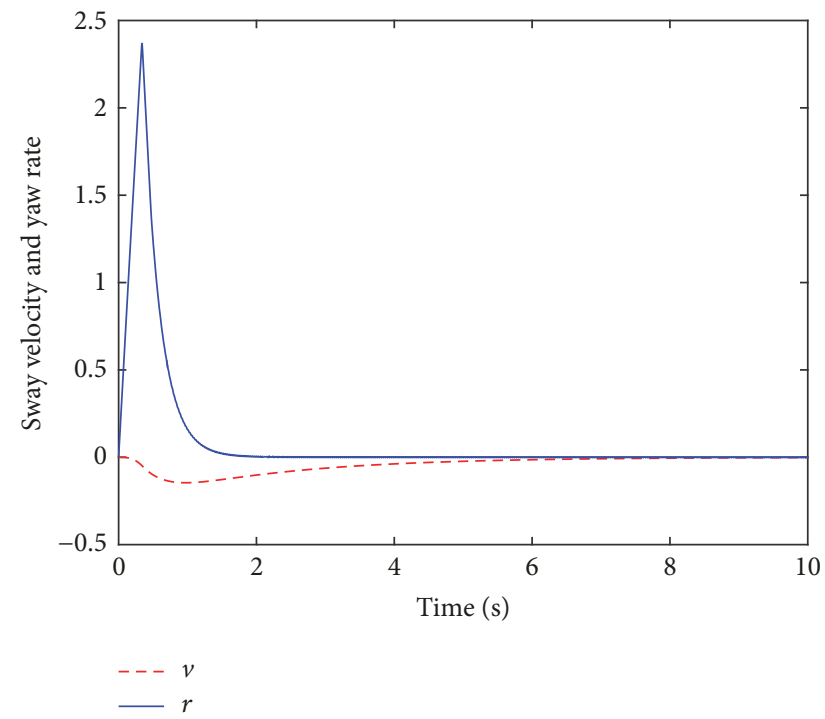

FIGURE 8: Sway velocity and yaw rate curve of USV when using the proposed controller.

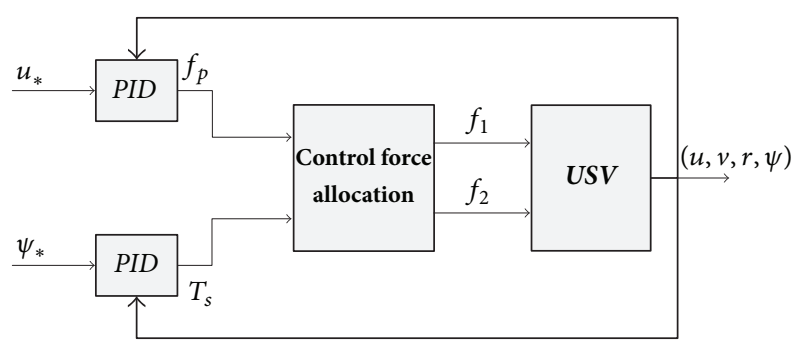

FIGURE 9: Structure of the classical PID controller.

\section{Conclusions}

In this article, the state error port-controlled Hamiltonian theory has been discussed. A novel controller based on state error port-controlled Hamiltonian approach is proposed in this paper for speed and heading angle tracking control of

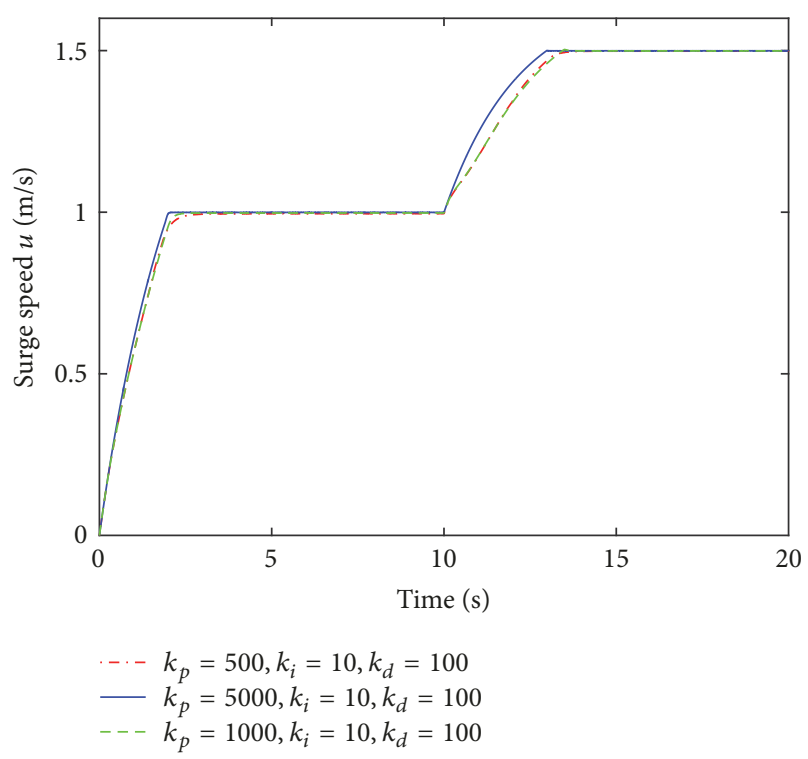

FIGURE 10: Surge speed curves with different parameters (classical PID controller).

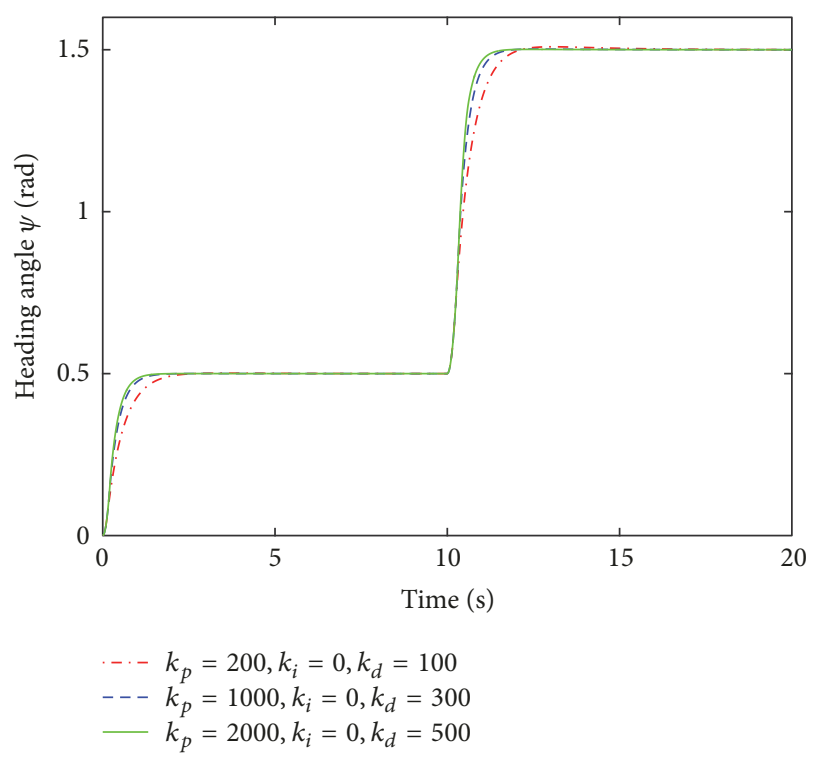

FIGURE 11: Heading angle curves with different parameters (classical PID controller).

underactuated USV. The desired state error port-controlled Hamiltonian structure is assigned to the closed-loop USV system which based on interconnection assignment and damping injection method. To realize the overall stability of the control, Lyapunov theory and La Salle's invariance principle are introduced to improve the clearance of physical meanings. Simulation results confirm the validity and stability of control algorithm. Compared with the classical PID controller, the designed controller has similar tracking and load disturbances attenuation performances. The designed controller has good steady state performance and simple structure. And the proposed controller provides an effective 


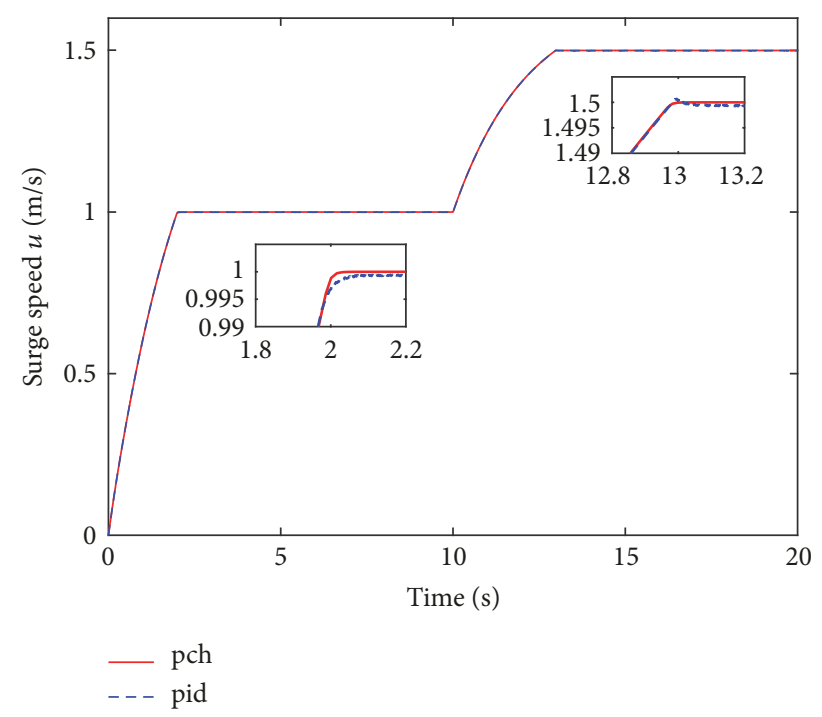

FIgURE 12: Surge Speed tracking curves when using different controllers.

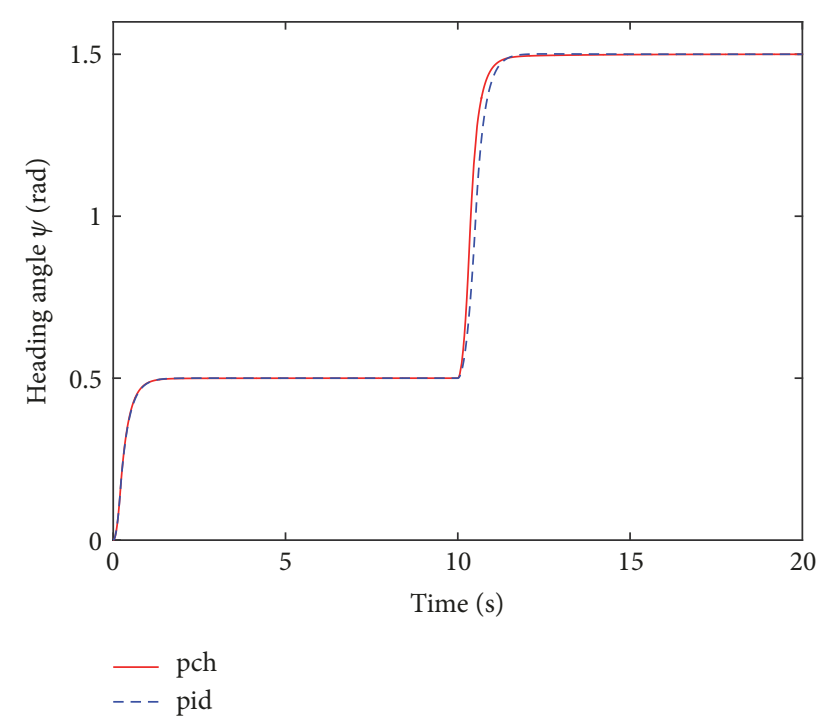

FIGURE 13: Heading angle tracking curves when using different controllers.

approach to analyze stability of the closed-loop system. The high simplification and portability of the controller make it a candidate choice for vast application in various region.

\section{Conflicts of Interest}

The authors declare that they have no conflicts of interest.

\section{Acknowledgments}

This work is partially supported by the National Natural Science Foundation of China (61573203) and National Key Research and Development Program of China (2016YFC1400802).

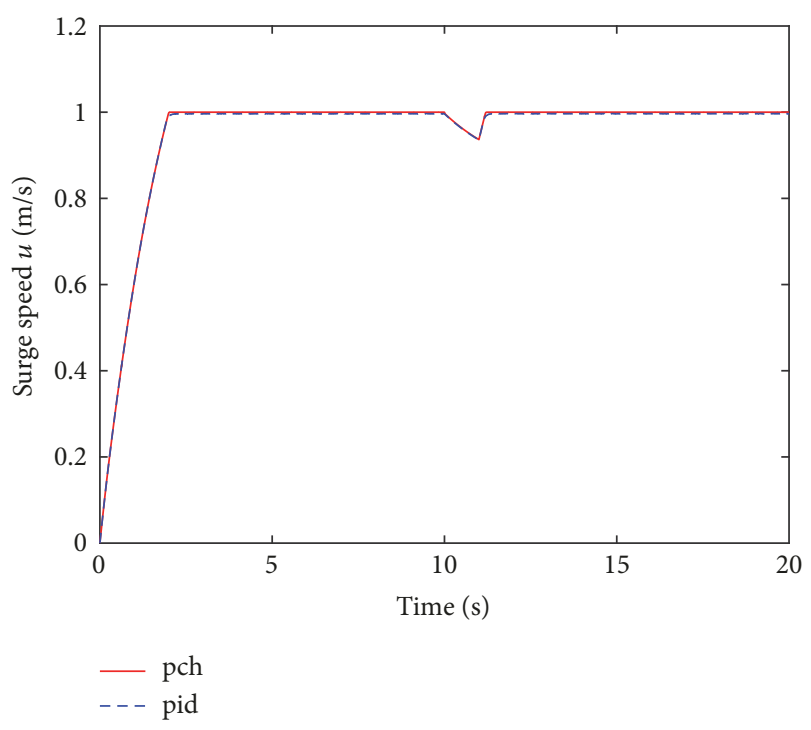

FIGURE 14: Surge speed response when using different controllers.

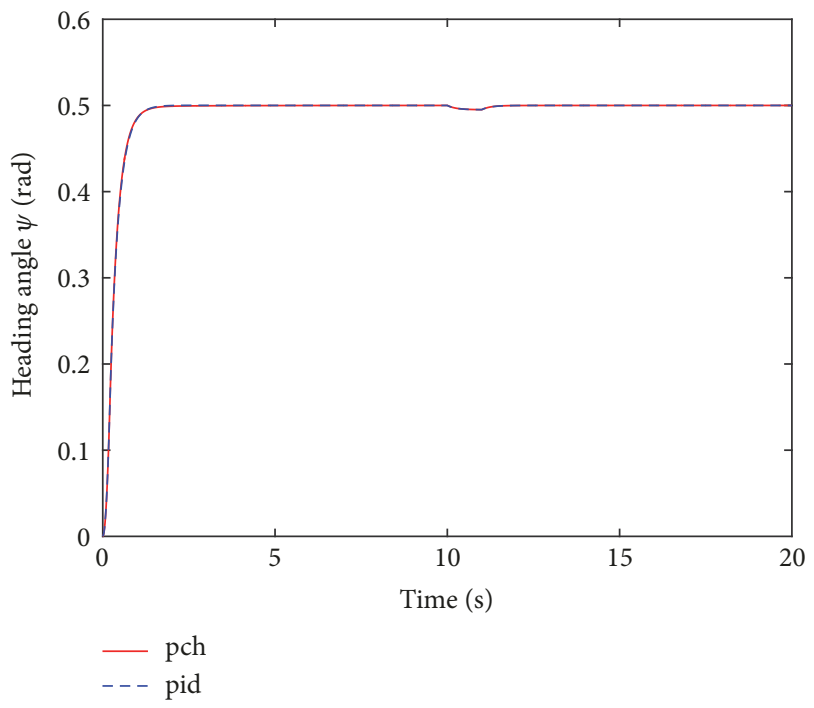

FIGURE 15: Heading angle response when using different controllers.

\section{References}

[1] Y. Ma, Y.Zhao, J. Diao, L. Gan, H. Bi, and J. Zhao, "Design of sailassisted unmanned surface vehicle intelligent control system," Mathematical Problems in Engineering, vol. 2016, Article ID 2958240, pp. 1-13, 2016.

[2] Z. Liu, Y. Zhang, X. Yu, and C. Yuan, "Unmanned surface vehicles: an overview of developments and challenges," Annual Reviews in Control, vol. 41, pp. 71-93, 2016.

[3] W. B. Klinger, I. R. Bertaska, K. D. Von Ellenrieder, and M. R. Dhanak, "Control of an unmanned surface vehicle with uncertain displacement and drag," IEEE Journal of Oceanic Engineering, pp. 1-19, 2016.

[4] C. R. Sonnenburg, Modeling, Identification, and Control of an Unmanned Surface Vehicle, Virginia Polytechnic Institute and State University, 2012. 
[5] C. R. Sonnenburg and C. A. Woolsey, "Modeling, identification, and control of an unmanned surface vehicle," Journal of Field Robotics, vol. 30, no. 3, pp. 371-398, 2013.

[6] Z. Dong, L. Wan, Y. Li, T. Liu, and G. Zhang, "Trajectory tracking control of underactuated USV based on modified backstepping approach," International Journal of Naval Architecture and Ocean Engineering, vol. 7, no. 5, pp. 817-832, 2015.

[7] V. D. Sean Kragelund, A. Monarrez, M. Hurban, and C. Khol, "Adaptive speed control for autonomous surface vessels," in Proceedings of the OCEANS 2013 MTS/IEEE San Diego Conference: An Ocean in Common, USA, September 2013.

[8] Y.-L. Liao, M.-J. Zhang, L. Wan, and Y. Li, “Trajectory tracking control for underactuated unmanned surface vehicles with dynamic uncertainties," Journal of Central South University, vol. 23, no. 2, pp. 370-378, 2016.

[9] Y.-L. Liao, Y.-M. Su, and J. Cao, "Trajectory planning and tracking control for underactuated unmanned surface vessels," Journal of Central South University, vol. 21, no. 2, pp. 540-549, 2014.

[10] H. Ashrafiuon, K. R. Muske, L. C. McNinch, and R. A. Soltan, "Sliding-mode tracking control of surface vessels," IEEE Transactions on Industrial Electronics, vol. 55, no. 11, pp. 40044012, 2008.

[11] N. E. Kahveci and P. A. Ioannou, "Adaptive steering control for uncertain ship dynamics and stability analysis," Automatica, vol. 49, no. 3, pp. 685-697, 2013.

[12] H. Yu, J. Yu, J. Liu, and Q. Song, "Nonlinear control of induction motors based on state error PCH and energy-shaping principle," Nonlinear Dynamics, vol. 72, no. 1-2, pp. 49-59, 2013.

[13] H. Yu, J. Yu, and Y. Wang, "Energy-shaping control of PMSM based on maximum output power and load torque observer," ICIC Express Letters, vol. 7, no. 1, pp. 241-246, 2013.

[14] R. Ortega, A. Van der Schaft, B. Maschke, and G. Escobar, "Interconnection and damping assignment passivity-based control of port-controlled Hamiltonian systems," Automatica, vol. 38, no. 4, pp. 585-596, 2002.

[15] R. Ortega and E. García-Canseco, "Interconnection and damping assignment passivity-based control: a survey," European Journal of Control, vol. 10, no. 5, pp. 432-450, 2004.

[16] A. S. K. Annamalai, R. Sutton, C. Yang, P. Culverhouse, and S. Sharma, "Robust adaptive control of an uninhabited surface vehicle," Journal of Intelligent \& Robotic Systems, vol. 78, no. 2, pp. 319-338, 2015.

[17] K. R. Muske, H. Ashrafiuon, G. Haas, R. McCloskey, and T. Flynn, "Identification of a control oriented nonlinear dynamic USV model," in Proceedings of the 2008 American Control Conference, ACC, USA, June 2008.

[18] I. R. Bertaska, J. Alvarez, A. Sinisterra et al., "Experimental evaluation of approach behavior for autonomous surface vehicles," in Proceedings of the ASME 2013 Dynamic Systems and Control Conference (DSCC '13), USA, October 2013.

[19] D. Mu, G. Wang, Y. Fan, and Y. Zhao, "Modeling and identification of podded propulsion unmanned surface vehicle and its course control research," Mathematical Problems in Engineering, Article ID 3209451, pp. 1-13, 2017.

[20] T. I. Fossen, Marine Control Systems : Guidance, Navigation and Control of Ships, Rigs and Underwater Vehicles, Marine cybernetics, 2002.

[21] I. B. Wilhelm, B. Klinger, J. Alvarez, and D. Karl, "Controller design challenges for waterjet propelled unmanned surface vehicles with uncertain drag and mass properties," in Procceedings of the EEE/MTS OCEANS Conference, San Diego, Calif, USA, 2013.

[22] Y. Liao, Y. Pang, and L. Wan, "Combined speed and yaw control of underactuated unmanned surface vehicles," in Proceedings of the 2010 2nd International Asia Conference on Informatics in Control, Automation and Robotics, CAR 2010, pp. 157-161, China, March 2010.

[23] K. D. Do and J. Pan, "Robust path-following of underactuated ships: Theory and experiments on a model ship," Ocean Engineering, vol. 33, no. 10, pp. 1354-1372, 2006. 


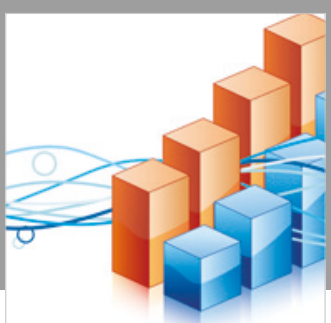

Advances in

Operations Research

\section{-n-m}
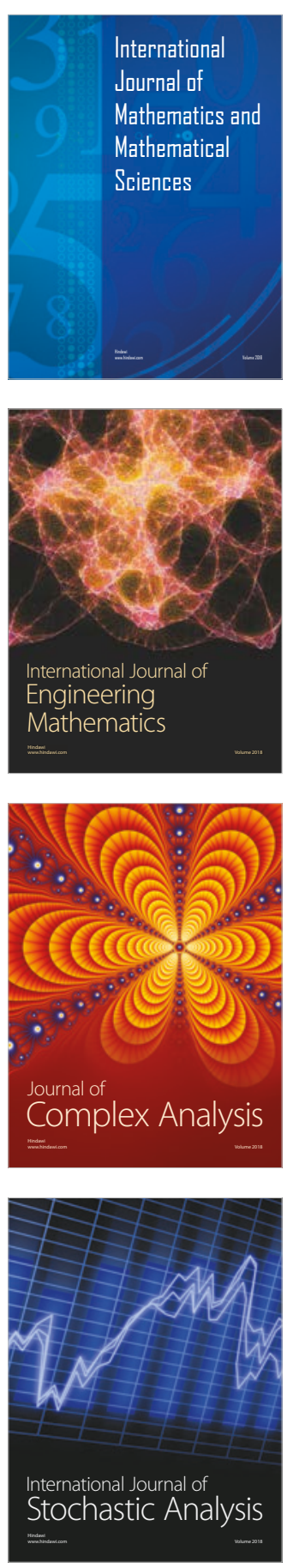
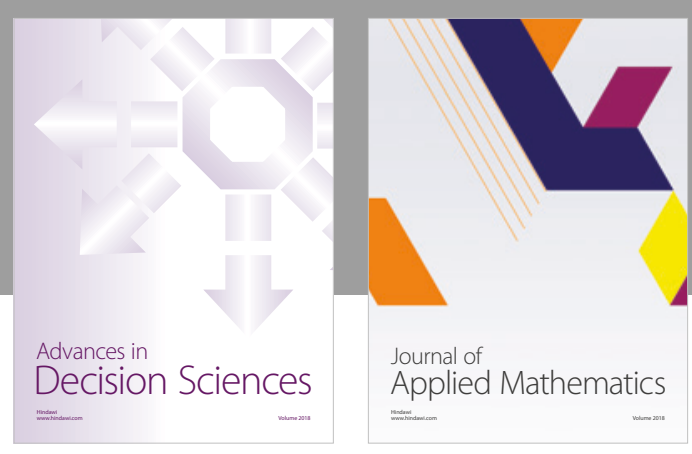

Journal of

Applied Mathematics
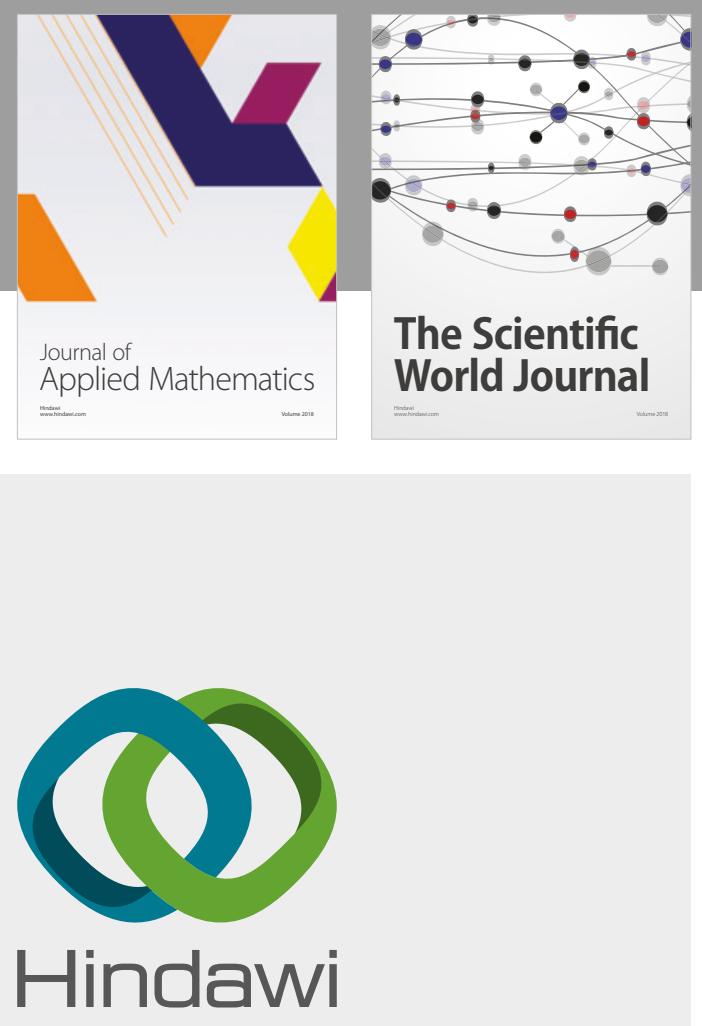

Submit your manuscripts at

www.hindawi.com

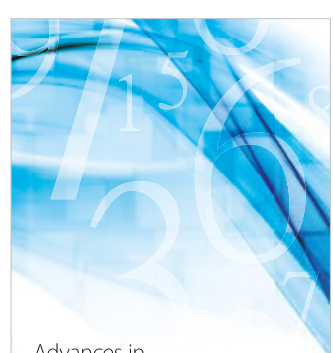

Advances in
Numerical Analysis
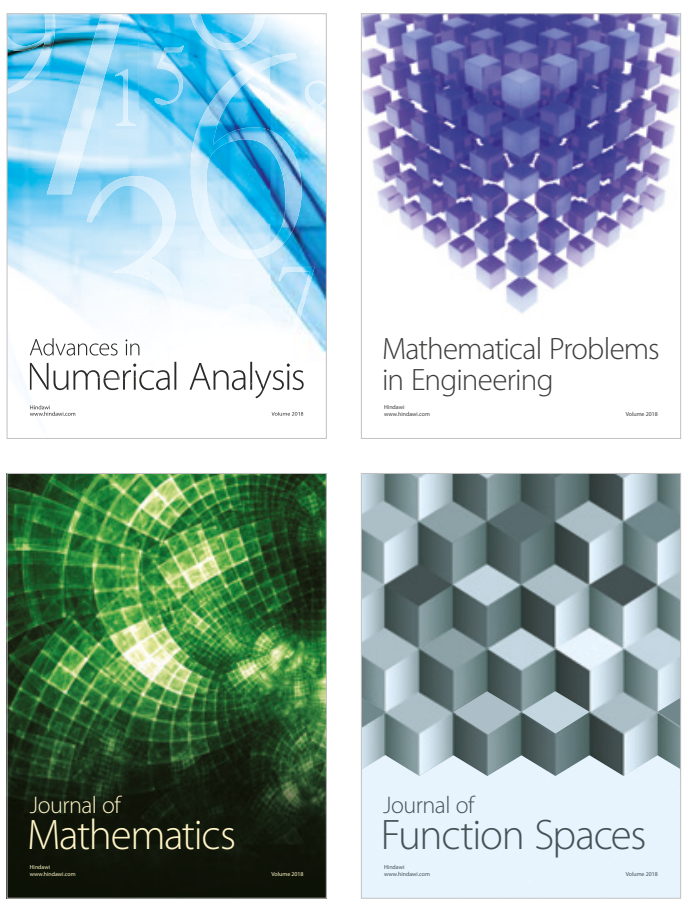

Mathematical Problems in Engineering

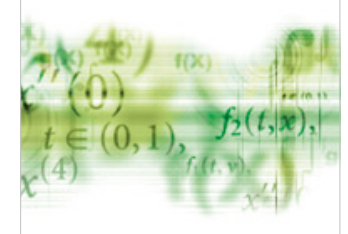

International Journal of

Differential Equations

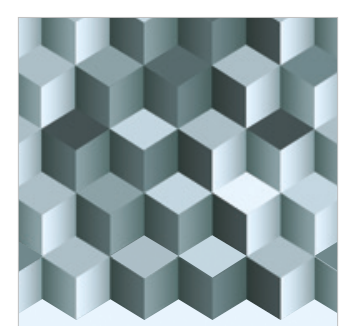

Journal of

Function Spaces

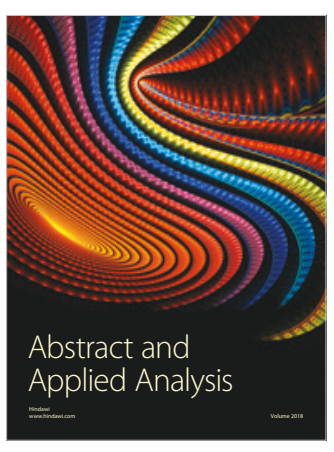

The Scientific

World Journal

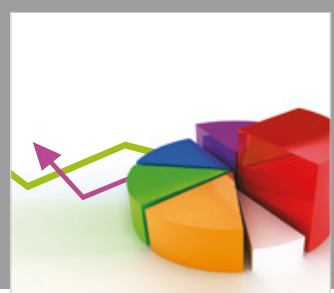

Journal of

Probability and Statistics
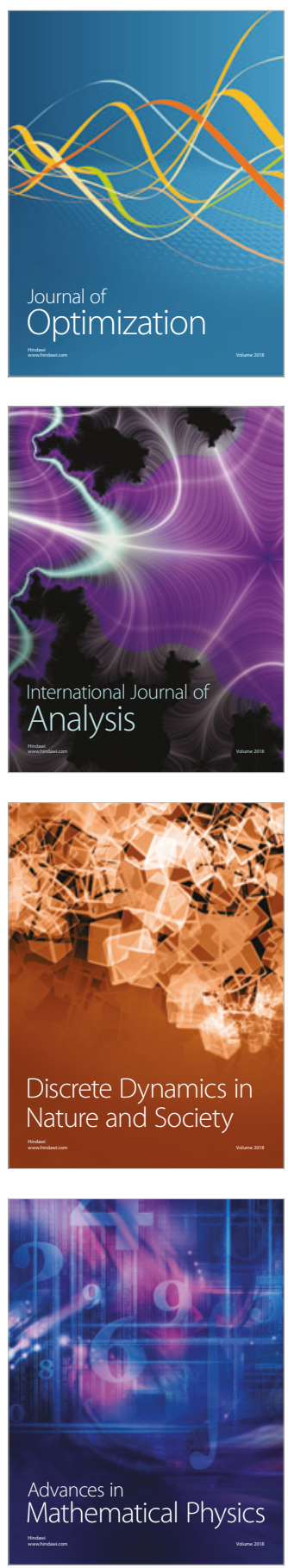